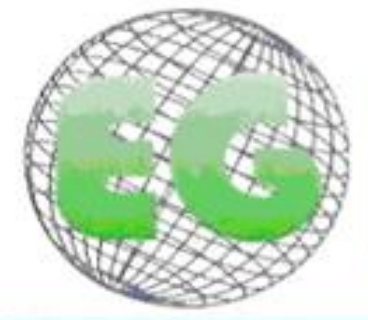

ISSN 1695-6141 No38

\title{
Internet y conducta sexual de riesgo para VIH/SIDA en jóvenes
}

Internet and sexual risk behavior for HIV/AIDS in young people

\section{*Valdez Montero, Carolina **Benavides Torres, Raquel Alicia ***González y González, Víctor **Onofre Rodríguez, Dora Julia ****Castillo Arcos, Lubia}

\begin{abstract}
*Estudiante de Doctorado en Ciencias de Enfermería. Facultad de Enfermería. Universidad Autónoma de Nuevo León, UANL E-mail: cavamontero@gmail.com **Profesor de la Universidad Autónoma de Nuevo León, FAEN, CIDICS, Monterrey, Nuevo León ***Ph.D. Instituto Autónomo de México, Departamento Académico de Computación, Distrito Federal. **** Doctora en Ciencias de Enfermería. Universidad Autónoma del Carmen. Ciudad del Carmen, Campeche. México.
\end{abstract}

Palabras clave: conducta sexual; riesgo; Internet; VIH; SIDA (Fuente: DeCs, Bireme). .Keywords: sexual behavior; risk; STIs; HIV; AIDS (Source: DeCs, Bireme)

\section{RESUMEN}

Objetivo: Determinar si el uso de material sexual en línea influye en la conducta sexual de riesgo para VIH/SIDA en los jóvenes universitarios. Se utilizaron conceptos de la Teoría Cognitiva Social.

Método: Diseño descriptivo correlacional, participaron 200 jóvenes universitarios, seleccionados por muestreo aleatorio sistemático $(k=11)$.

Resultados: Los jóvenes que usaron material sexual en línea en medios ricos para masturbarse $\left(r_{s}=\right.$ .34), excitarse $\left(r_{s}=.29\right)$, estimularse $\left(r_{s}=.29\right)$, buscar una aventura $\left(r_{s}=.30\right)$, conocer gente $\left(r_{s}=.27\right)$, imágenes $\left(r_{s}=.14\right)$ y cibersexo $\left(r_{s}=.25\right)$ mostraron mayor conducta sexual de riesgo para VIH/SIDA ( $p$ $<.01)$. El uso de material sexual en línea para masturbarse $\left(R^{2}=6.4 \%, F_{[1,189]}=12.80, p<.001\right)$, buscar una aventura $\left(R^{2}=4.8 \%, F_{[1,189]}=9.56, p<.01\right)$, conocer gente $\left(R^{2}=5.9 \%, F_{[1,189]}=11.88, p<\right.$ $.01)$ y tener cibersexo $\left(R^{2}=4.1 \%, F_{[1,189]}=8.07, p<.01\right)$ presentó un efecto positivo y significativo en la conducta sexual de riesgo para $\mathrm{VIH} / \mathrm{SIDA}$.

Conclusiones: El uso de material sexual en línea influye en la conducta sexual de riesgo para VIH/SIDA.

\section{ABSTRACT}

Objective: To determine whether the use of online sexual material influences sexual risk behavior for HIV / AIDS in young university students. Concepts of Social Cognitive Theory were used. 
Methods: A descriptive correlational design, involving 200 university students selected by systematic random sampling $(k=11)$.

Results: Young people who used sexual material online rich media to masturbation $\left(r_{s}=.34\right)$, arousal $\left(r_{s}\right.$ $=29)$, stimulation $\left(r_{s}=29\right)$, adventure $\left(r_{s}=30\right)$, meeting people $\left(r_{s}=.27\right)$, images $\left(r_{s}=.17\right)$ and cybersex $\left(r_{s}=.25\right)$ showed greater sexual risk behavior for HIV / AIDS ( $\left.p<.01\right)$. The use of sexual material online for masturbation $\left(R^{2}=6.4 \%, F_{[1,189]}=12.80, p<.001\right)$, seeking adventures $\left(R^{2}=4.8 \%, F_{[1,189]}=9.56, p\right.$ $<.01)$, meeting people $\left(R^{2}=5.9 \%, F_{[1,189]}=11.88, p<.01\right)$ and have cybersex $\left(R^{2}=4.1 \%, F_{[1,189]}=\right.$ $8.07, p<.01$ ) had a significant positive effect on behavior sexual risk for HIV/AIDS.

Conclusions: The use of online sexual material influences sexual risk behavior for HIV/AIDS.

\section{INTRODUCCIÓN}

El Síndrome de Inmunodeficiencia Adquirida (SIDA) es un problema de salud pública de carácter transmisible que afecta principalmente a poblaciones vulnerables. Los jóvenes son considerados población vulnerable debido a que comienzan a tomar decisiones y a reconocer su identidad, en esta etapa de cambios puede llegar a ser un periodo vulnerable para que los jóvenes adopten una conducta sexual de riesgo para adquirir Infecciones de Transmisión Sexual (ITS) o aún más grave el Virus de la Inmunodeficiencia Humana (VIH)/SIDA.

Se estima que a nivel mundial cada día se contagian de SIDA 2,500 jóvenes y que existen más de 5.7 millones infectados por $\mathrm{VIH}{ }^{(1)}$. En América Latina y el Caribe se encuentran viviendo con VIH más de 740.000 jóvenes entre los 15 y 24 años (2). Específicamente en México el $98.9 \%$ de los casos diagnosticados de SIDA en los jóvenes fue por transmisión sexual ${ }^{(3)}$.

Existen diversos factores de riesgo para que los jóvenes adopten conductas sexuales riesgosas, uno de ellos es la exposición a contenido sexual en los medios de comunicación ${ }^{(4)}$. El Internet es el medio de comunicación más usado en estos días y tiene como fin comunicar, socializar y entretener a los usuarios. En México existen más de 45.1 millones de usuarios del Internet de los cuales el $43 \%$ de estos son jóvenes ${ }^{(5)}$. Asimismo, se estima que siete de cada diez jóvenes de 12 a 19 años, se conectan al Internet para fines de comunicación e interacción; de éstos el 90\% envía o recibe e-mail, el $75 \%$ envían o reciben mensajes instantáneos, el $61 \%$ accede, crea y/o mantiene redes sociales y el $14 \%$ lo utiliza para la búsqueda de pareja ${ }^{(6)}$.

Cooper, Scherer, Boies y Gordon ${ }^{(7)}$ refieren que el Internet se ha convertido en una potente conexión entre los jóvenes y el material erótico y sexual, lo que puede favorecer el inicio precoz de las relaciones sexuales y por consiguiente mayores conductas de riesgo para ITS-VIH/SIDA. Diversos estudios han demostrado que los jóvenes que pasan mayor tiempo en el Internet tienen mayores probabilidades de ver contenido de tipo sexual, como pornografía, mantener conversaciones sexuales, masturbarse y/o practicar cibersexo $(8,9,10,11,12)$

Algunos autores $(4,13,14,15)$ han utilizado la Teoría Cognitiva Social (TCS) como fundamento de estudios relacionados con el uso del Internet y la conducta sexual. Los autores mencionan que los comportamientos riesgosos son adquiridos y reforzados a través de interacciones sociales y se aprenden por medio de la observación y la imitación de las acciones de otros. Asimismo, si se percibe que el resultado de la conducta que se aprendió por medio de la observación es satisfactorio, entonces es muy probable que la persona repita esta conducta. Con relación a esto, el consultar o 
interactuar con material sexual en línea como ver pornografía, desnudos, tener conversaciones sexuales y/o cibersexo puede provocar excitación en los jóvenes lo que pudiera desencadenar conductas sexuales de riesgo para adquirir VIH/SIDA como el sexo anal, oral y/o vaginal sin protección y con múltiples parejas sexuales.

En el mismo sentido, Melamud et al ${ }^{(16)}$, consideran que el Internet puede convertirse en un riesgo y refieren que éste es la "nueva epidemia del siglo XXI". Es por esto que la profesión de Enfermería ha implementado nuevas formas para promover la salud y prevenir las enfermedades. El término "Nursing Informatics" o informática en enfermería es de especial relevancia ya que a través de la tecnología computacional las enfermeras brindan servicios, proporcionan educación y realizan investigaciones $^{(17)}$. Hasta donde se indagó, en México no se han encontrado estudios que hayan investigado el efecto del uso de material sexual en línea en la conducta sexual de riesgo para adquirir VIH/SIDA en jóvenes. Por tal motivo el objetivo general de este estudio fue determinar si el uso de material sexual en línea influye en la conducta sexual de riesgo en jóvenes universitarios.

\section{MÉTODO}

En el presente estudio se utilizó un diseño descriptivo correlacional de tipo transversal ${ }^{(18)}$. La población se conformó por 200 jóvenes entre 18 a 25 años de ocho facultades de una universidad privada de Monterrey, Nuevo León y fueron seleccionadas por medio de un muestreo aleatorio sistemático $(k=11)$ proporcional al género y al número de estudiantes por facultad. La muestra fue estimada para una prueba de correlación con un coeficiente de .20, un nivel de confianza del $95 \%$ y una potencia del $90 \%$.

\section{Instrumentos}

Se utilizó una cédula de datos sociodemográficos para describir a los participantes del estudio en la que se incluyó: edad, sexo y estado civil. Para medir el uso de material sexual en línea se utilizó el instrumento de Gonsalves ${ }^{(15)}$ compuesto por 45 preguntas que evalúan siete actividades sexuales en línea: masturbación, excitación, estimulación, aventura, conocer gente, imágenes y cibersexo. Este instrumento ha mostrado confiabilidad aceptable, Alpha de Cronbach de .72 a $.90^{(15)}$. Para medir la conducta sexual de riesgo para VIH/SIDA se utilizó el instrumento de Comportamiento Sexual de la Encuesta de Salud Estudiantil ${ }^{(19)}$. Este consta de 31 preguntas que evalúan la conducta sexual de riesgo para $\mathrm{VIH} / \mathrm{SIDA}$, este instrumento, en la prueba test-retest ha reportado valores por arriba de $.64^{(20)}$ y se ha reportado validez de convergencia con coeficientes aceptables entre .76 y $.88^{(21)}$.

\section{Procedimiento}

Para la realización del presente estudio se solicitó la aprobación de las comisiones de Investigación y Ética de la Facultad de Enfermería de la Universidad Autónoma de Nuevo León (UANL). Se pidió la autorización de los directivos de la universidad privada donde se realizó el estudio. Posteriormente se acudió a las puertas principales de las facultades ubicándose de lado derecho, después se inició el conteo de 1 en 11 con un contador de personas comenzando con la primera persona que pisara la entrada de la puerta contando de derecha a izquierda, a la persona seleccionada se le explicó el propósito del estudio, se les invitó a participar en el mismo y se les pidió que firmaran el consentimiento informado, se les informó que la 
encuesta se les enviaría por correo electrónico para que la contestaran desde sus hogares o lugar de preferencia, se les dio una semana para que la respondieran y a su vez se les estuvieron enviando recordatorios cada tercer día hasta que fuera contestada la encuesta. El llenado de los cuestionarios se realizó a través del portal Surveymonkey. Se les pidió a los participantes que sus respuestas fueran las más honestas posibles y se les aseguró que sus respuestas se mantendrían en absoluta confidencialidad. Este estudio se apegó al Reglamento de la Ley General de Salud en Materia de Investigación para la Salud ${ }^{(22)}$.

\section{Estrategia de análisis de datos}

Los datos fueron analizados en el paquete estadístico Statistical Package for the Social Sciences (SPSS) versión 18 para Windows ${ }^{\circledR}$. Previo al análisis, se generaron índices para cada una de las escalas y sub-escalas de los instrumentos utilizados. Para describir las variables del estudio se utilizó la estadística descriptiva, como medias, mínimos y máximos para las variables continuas y frecuencias y porcentajes para las variables categóricas. Previo al análisis de los datos, se procedió a verificar si las variables contaban con distribución normal por medio de la prueba de Kolmogorov-Smirnov (K-S) con corrección de Lilliefors. Debido a que la mayoría de las variables no presentaron distribución normal se utilizó la estadística no paramétrica para analizar el objetivo del estudio y se utilizó prueba de correlación de Spearman. Asimismo se utilizó Modelos de Regresión Lineal Simple.

\section{RESULTADOS}

La edad media de los jóvenes universitarios fue de 20.6 años ( $D E=1.83$ ), predominando el género masculino (53\%). En relación al uso del Internet, la mayoría mencionó tener acceso a una computadora (97\%), de ellos se encontró que el $51.5 \%$ ha utilizado una computadora para consultar y/o interactuar con material sexual en línea y que en un día normal utilizan de 10 a 120 minutos para esta actividad $(M=$ $44.88, D E=24.67)$. También mencionaron que se encuentran solos(as) cuando usan material sexual en línea (53.5\%), de los cuales el 64.1\% tenía entre 14 y 18 años la primera vez que consultaron y/o interactuaron con material sexual en línea. La mayor parte tiene acceso a este tipo de material a través de páginas de Internet (64\%).

En relación a la conducta sexual de riesgo para $\mathrm{VIH} / \mathrm{SIDA}$, se encontró que el $74.7 \%$ de los jóvenes ha tenido sexo vaginal, el $59 \%$ sexo oral y el $27.9 \%$ sexo anal. La media de edad de la primera relación sexual fue de 17.07 años $(D E=1.98)$, en promedio han tenido más de dos parejas sexuales $(M=2.45, D E=2.13)$ y la mayoría reporta que no planean sus relaciones sexuales (56\%). En cuanto al uso del condón, se encontró que el $37.6 \%$ no utilizó el condón cuando tuvo sexo vaginal, el $87.3 \%$ cuando tuvo sexo oral y el $50.9 \%$ cuando tuvo sexo anal por primera vez. El $32.4 \%$ han tenido más de 9 encuentros sexuales en los últimos 12 meses y el $4.3 \%$ tuvo relaciones sexuales con personas de su mismo sexo. El instrumento total presentó una media de $35(D E=18.89 ;$ Max. = 71), lo cual indica que los jóvenes están teniendo una conducta sexual de riesgo para adquirir VIH/SIDA.

Para cumplir con el objetivo del presente estudio se encontró que existe una correlación significativa entre el uso de material sexual en línea y la conducta sexual de riesgo para $\mathrm{VIH} / \mathrm{SIDA}$ (tabla 1). La mayoría de los coeficientes fueron moderados, excepto para la escala de imágenes que mostró un coeficiente débil. Por lo que, se puede decir que aquellos jóvenes que usaron material sexual en línea para realizar 
actividades sexuales como masturbarse, excitarse, estimularse, buscar una aventura, conocer gente, compartir imágenes de actos sexuales y tener cibersexo mostraron mayor conducta sexual de riesgo para VIH/SIDA $(p<.01)$.

Tabla 1. Matriz de Correlación de Spearman entre el uso de material sexual en línea y la conducta sexual de riesgo para VIH/SIDA

\begin{tabular}{ll}
\hline UMSL & CSR para VIH/SIDA \\
\hline Masturbación & $.34^{\star \star}$ \\
Excitación & $.29^{\star *}$ \\
Estimularse & $.29^{\star \star}$ \\
Aventura & $.30^{\star *}$ \\
Conocer gente & $.27^{\star \star}$ \\
Imágenes & $.14^{\star}$ \\
Cibersexo & $.25^{\star *}$ \\
\hline
\end{tabular}

Nota: UMSL = Uso de Material Sexual en Línea. CSR = Conducta Sexual de Riesgo. ${ }^{*} p<.05 ;{ }^{* *} p<.01$.

Asimismo, se realizaron pruebas de regresión lineal simple. En la tabla 2 se puede observar que el uso de material sexual en línea influye en la conducta sexual de riesgo para VIH/SIDA. Se encontró que el uso de material sexual en línea para realizar actividades sexuales en línea como: masturbarse, buscar una aventura, conocer gente y tener cibersexo presentó un efecto positivo y significativo en la conducta sexual de riesgo para VIH/SIDA.

Tabla 2. Regresión Lineal Simple de uso de material sexual en línea con conducta sexual de riesgo para $\mathrm{VIH} / \mathrm{SIDA}$

\begin{tabular}{lllll}
\hline Modelos UMSL & $B$ & Error & Beta & Valor de \\
& & Estándar & & $P$
\end{tabular}

\begin{tabular}{llllll}
\hline 1 & Masturbación & .30 & .09 & .25 & .00 \\
\hline 2 & Excitación & .30 & .15 & .15 & .04 \\
\hline 3 & Estimularse & .15 & .11 & .09 & .20 \\
\hline 4 & Aventura & .44 & .14 & .22 & .00 \\
\hline 5 & Conocer gente & .38 & .11 & .24 & .00 \\
\hline 6 & Imágenes & .43 & .29 & .11 & .14 \\
\hline 7 & Cibersexo & .40 & .14 & .20 & .00 \\
\hline
\end{tabular}

Nota: $\mathrm{UMSL}=$ Uso de Material Sexual en Línea.

\section{DISCUSIÓN}

En función de los hallazgos encontrados para el cumplimiento del objetivo del presente estudio, se encontró que más de la mitad de los jóvenes han utilizado material sexual en línea. Esto puede deberse a que los jóvenes son quienes utilizan en mayor medida el Internet a comparación con otros grupos de edad (23). Este porcentaje elevado se podría explicar porque al tener un uso elevado del Internet tienen mayor exposición al uso de material sexual en línea. También se encontró que la mayoría de los jóvenes realizan esta actividad cuando se encuentran solos y siendo menores de edad, lo cual es ilegal. Esto concuerda con lo reportado por Cooper (24) quien refiere que una de las características de la actividad sexual en línea es la accesibilidad y el anonimato; asimismo Ybarra y Mitchell ${ }^{(25)}$ refieren que los 
adolescentes mienten acerca de su edad para visitar lugares con material sexual en línea.

En cuanto a la conducta sexual de riesgo para VIH/SIDA, se encontró que la mayoría de los jóvenes ya habían iniciado su vida sexual y que este inició se dio antes de que cumplieran la mayoría de edad. Lo cual concuerda con lo reportado por el CENSIDA (26) que menciona que el grupo que tiene mayor riesgo de adquirir el VIH/SIDA es el de 10 a 29 años. Un dato relevante que no había sido explorado con anterioridad es el uso del condón durante el sexo oral, en este estudio se encontró que la mayoría de los jóvenes no lo utilizó ni en su primera, ni la última experiencia con este tipo de sexo. Este resultado puede deberse a que la mayoría de los jóvenes no saben que también pueden infectarse del VIH por medio del sexo oral ${ }^{(27)}$, además de que ellos no creen que el sexo oral es un acto sexual ${ }^{(28)}$. También se encontró que la mayoría de los jóvenes utilizan el condón de manera inconsistente y que tienen múltiples parejas sexuales, lo cual concuerda con diversos estudios ${ }^{(29,30,31,32,33)}$. Se encontró que gran parte de los jóvenes que participaron en el estudio no planeaban sus relaciones sexuales, lo cual es congruente con su etapa de desarrollo ya que a esta edad aún son muy impulsivos y no controlan sus actos ${ }^{(34)}$.

Asimismo, en el presente estudio se encontró que a mayor uso de material sexual en línea, mayor es la conducta sexual de riesgo para VIH/SIDA. Esto es similar a lo propuesto por diversos autores ${ }^{(13,14,15,35)}$ quienes utilizaron la Teoría Cognitiva Social y sugieren que las conductas sexuales de riesgo para VIH/SIDA pueden ser adquiridas y reproducidas en sus comportamientos, a través de la imitación y copia de actos de modelos sexuales que se encuentran en el Internet.

A su vez, se determinó que el uso de material sexual en línea tiene un efecto significativo en la conducta sexual de riesgo para VIH/SIDA. De esto, se puede decir que los jóvenes pueden sentir mayor placer al usar material sexual en línea y que estos pueden tener un mayor impacto en su aprendizaje ya que pudieran estar más motivados a modelar lo observado en línea. Asimismo, Bandura ${ }^{(36)}$ refiere que los individuos tienen la capacidad de aprender observando. Por lo que se puede inferir, que los jóvenes pudieron incrementar su aprendizaje sobre sus conductas sexuales de riesgo para $\mathrm{VIH} / \mathrm{SIDA}$ y que los pudieron haber reproducido en sus comportamientos sexuales ${ }^{(37)}$.

En cuanto al diseño, se puede decir que resultó apropiado para llevar a cabo el presente estudio, sin embargo, el muestreo aleatorio sistemático que implica el uso de un cuenta personas, requiere de mayor personal. Una limitante fue en relación a la sensibilidad de la temática, debido a que la sexualidad aún es considerada un tabú en nuestra sociedad, si bien se utilizó la plataforma por Internet, no se evaluó la complacencia social. En ese mismo orden de ideas, cabe señalar que algunos conceptos relacionados con la sexualidad requieren ser clarificados ya que se presentaron inconsistencias en las preguntas de sexo oral, debido a que no se valoró si los participantes consideran el sexo oral como una conducta sexual. Por último, debido a que el estudio fue de tipo transversal y los datos fueron recolectados en una Institución de Educación Privada, la generalización de los mismos debe de hacerse a jóvenes con características similares sin establecer una relación causa-efecto.

Para futuros estudios se recomienda incluir adolescentes de temprana edad (10 a 14 años) para realizar seguimientos de estas variables a lo largo del tiempo y establecer una relación causa efecto. Se recomienda continuar con el uso de plataformas en Internet como el SurveyMonkey ya que estas son de bajo costo y permiten además 
dar una mayor confidencialidad al sujeto y al exportar los datos se evita error en la captura. También, sería de interés indagar la relación que existe entre el uso de material sexual en línea y comportamientos sexuales de riesgo para VIH/SIDA más específicos, por ejemplo: número de parejas sexuales. Sería de especial relevancia realizar un estudio similar en población de hombres que tienen sexo con hombres, ya que la mayor parte de ellos establece relaciones por medio del Internet. Con base a los hallazgos de este estudio se sugiere realizar intervenciones dirigidas a niños, adolescentes y jóvenes para establecer medidas de higiene sexual en los medios electrónicos, principalmente en el Internet.

\section{CONCLUSIONES}

Se puede concluir que los jóvenes utilizan material sexual en línea desde temprana edad y que a la mayoría de ellos les provoca placer observar material sexual en donde se involucran jóvenes de su edad. La mayoría de los jóvenes reportaron una conducta sexual de riesgo ya que la mayoría de los participantes han tenido sexo vaginal, anal u oral en los últimos doce meses, la edad de inicio de las relaciones sexuales fue de 17 años en promedio, más de la mitad reportó no haber utilizado el condón de manera consistente y un tercio de estos han tenido sexo con más de cuatro parejas sexuales, lo cual los pone en alto riesgo de adquirir una ITS-VIH/SIDA. Asimismo, el uso de material sexual en línea influye en la conducta sexual de riesgo para VIH/SIDA. Por último se puede decir que los hombre consultan/interactúan en mayor medida con material sexual en línea que las mujeres. Los hallazgos encontrados del estudio confirman lo planteado por la Teoría Cognitiva Social.

\section{REFERENCIAS BIBLIOGRÁFICAS}

1. Organización Mundial de la Salud. Los accidentes de tránsito, el suicidio y las afecciones maternas figuran entre las principales causas de muerte de los jóvenes. 2009 [citado 17 agosto 2012] Disponible en: http://www.who.int/mediacentre/news/releases/2009/adolescent mortality 2009091 $1 / \mathrm{es} /$

2. Fondo de las Naciones Unidas para la Infancia (Unicef). VIH/SIDA infancia y adolescencia en América Latina y el Caribe. 2005 [citado 8 febrero 2013] Disponible en: http://www.unicef.org/lac/infanciadolescencia.pdf

3. 26. Centro Nacional para la Prevención y Control del VIH/SIDA. Casos nuevos y acumulados de SIDA en jóvenes de 15 a 29 años, por categoría de transmisión y sexo. 2009 [citado 5 febrero 2011] Disponible en: http://www.censida.salud.gob.mx/descargas/2009/cifras/Nov2009/05 casoNuevAcu mJoven.pdf

4. Escobar C, Tortolero S, Markham C, Low B, Eitel P, Thickstum P. Impact of the media on adolescent sexual attitudes and behaviors. Journal of the American Academy of Pediatrics 2005; 116 (1): 303-326.

5. Asociación Mexicana de Internet. Hábitos de los usuarios de Internet en México 2013. [citado 17 noviembre 2013] Disponible en: http://www.amipci.org.mx/?P=editomultimediafile\&Multimedia=348\&Type $=1$

6. Asociación Mexicana de Internet. Hábitos de los usuarios de Internet en México 2011. [Citado $15 \quad$ octubre 2013$]$ Disponible $\quad$ en: http://www.amipci.org.mx/?P=editomultimediafile\&Multimedia=102\&Type =1

7. Cooper A, Scherer C., Boies S, Gordon B. Sexuality on the Internet: from sexual exploration to pathological expression. Professional Psychology: Research and Practice 1999; 30 (2): 154-164 
8. McFarlane M, Bull S, Rietmeijer C. The Internet as a newly emerging risk environment. The Journal of the American Medical Association 2000; 284 (4): 443446.

9. Toomey K, Rothenberg R. Sex and cyberspace-virtual networks leading to high-risk sex. The Jorunal of the American Medial Association 2000; 284(4): 485-487.

10. Wolak D, Finkelhor D, Mitchell K. Internet-initiated sex crimes against minors: Implications for prevention based on findings from a national study. Journal of Adolescent Health 2004; 11: 424.

11. Griffiths, M. Sex on the Internet: Observations and implications for Internet sex addiction. The Journal of Sex Research 2001; 38 (4): 333.

12. Peter J, Valkenburg P. Adolescents' exposure to sexually explicit material on the Internet. Communication Research 2006; 33: 178-204.

13. Adebayo D, Udegbe I, Sunmola A. Gender, Internet use, and sexual behavior orientation among young Nigerians. Cyberpsychology \& Behavior 2006; 9 (6): 242252.

14. Courtney $P$. Internet use and its effect on sexual behavior in traditional. (Tesis doctoral). The University of Florida, United States. Disponible en: Dissertations \& Theses: Full Text database; 2008.

15. Gonsalves V. Exploring online sexually explicit material: What is the relationship to sexual coercion? (Tesis doctoral). The University of Nebraska, United States, Linconln; 2010.

16. Melamud A, Otero P, Nasanovsky J, Stechina D, Goldfarb G, Svetliza J. et ál. Los niños, sus padres, Internet y los pediatras. Archivos Argentinos de Pediatría 2007; 71 (4): 105-368.

17. Nursing informatics. Expore health careers.org. 2008 [citado 8 abril 2011] Disponible en:

http://explorehealthcareers.org/en/Career/91/Nursing_Informatics

18. Burns N, Grove S. The practice of nursing research. Appraisal, synthesis, and generation of evidence (6th ed.). St. Louis: Elsevier Saunders; 2009.

19. Marín B, Coyle K, Gomez C, Carvajal S, Kirby D. Older boyfriends and girlfriends increase risk of sexual initiation in young adolescents. Journal of Adolescent Health 2000; 27: 409-418.

20. Motañez M. Normas subjetivas para VIH/SIDA y conducta sexual en adolescentes. (Tesis de maestría). Universidad Autónoma de Nuevo, León. México; 2008.

21. Valle M. Modelo motivacional para la conducta sexual de riesgo para VIH/SIDA en jóvenes (Tesis Doctoral). Universidad Autónoma de Nuevo León. México; 2011.

22. Secretaría de Salud. Reglamento de la Ley General de Salud en Materia de Investigación para la Salud. 1987 [citado 27 mayo 2011]_Disponible en: http://www.salud.gob.mx/unidades/cdi/nom/compi/rlgsmpsam.html

23. Instituto Nacional de Estadística y Geografía. Estadísticas a propósito del día mundial de Internet datos nacionales. 2013 [citado 19 noviembre 2013] Disponible en:

http://www.inegi.org.mx/inegi/contenidos/espanol/prensa/Contenidos/estadisticas/2 013/internet0.pdf

24. Cooper A. Sex \& the internet: a guidebook for clinicians. New York: BrunnerRoutledge; 2002.

25. Ybarra M, Mitchell K. Exposure to Internet pornography among children and adolescents: A national survey. Cyber Psychology \& Behavior 2005; 8: 473-486.

26. Vigilancia Epidemiológica de casos de VIH/SIDA en México registro nacional de casos de SIDA actualización al cierre de 2013. [citado 27 enero 2014] Disponible en:

http://www.censida.salud.gob.mx/descargas/epidemiologia/RN CIERRE 2013.pdf 
27. Salazar L, Crosby R, DiClemente R, Wingood G, Rose E, McDermott-Sales J, Caliendo A. African-American female adolescents who engage in oral, vaginal and anal sex: "doing it all" as a significant marker for risk of sexually transmitted infection. Aids and Behavior 2009; 13 (1): 85-93.

28. Remez L. Oral sex among adolescents: is it sex or is it abstinences. Family Planning Perspectives 2000; 32 (6): 298-299.

29. Aymerich A, Planes P, Gras P, Vila C. Relaciones heterosexuales e intenciones de conductas de riesgo en estudiantes de bachillerato. Anuario de Psicología Clínica y de la Salud 2008; 4: 63-71.

30. Empelen P, Kok G. Action-specific cognition of planned and preparatory behaviors of condom use among dutch adolescents. Arch. Sexual Behavior 2008; 37: 626640.

31. Lohman B, Billings A. Protective and risk factors associated with adolescent boys' early sexual debut and risk sexual behavior. Journal Youth adolescence 2008; 37: 723-735.

32. Mueller TE, Castaneda CA, Sainer S, Martinez D, Herbst JH, Wilkes AL, Villarruel AM. The implementation of a culturally based HIV sexual risk reduction program for latino youth in a Denver area high school. AIDS Education and Prevention 2009; 21 : 164-170.

33. Piña JA, Robles S. Psicología y VIH/SIDA en México: su prevención con base en un modelo Psicológico de salud biológica. Revista de Psicopatología y Psicología Clínica 2005; 10 (1): 71-83.

34. Vargas T, Henao J, González C. Toma de decisiones sexuales y reproductivas en la adolescencia. Acta Colombiana de Psicología 2007; 10 (1): 49-63.

35. Goodson P, McCormick D, Evans A. Searching for sexually explicit materials on the Internet: An exploratory study of college students' behavior and attitudes. Archives of Sexual Behavior 2001; 30 (2): 101.

36. Bandura A. Social foundations of thought and action: A social cognitive theory. Englewood Cliffs, New Jersey: Prentice-Hall; 1986.

37. McFarlane M, Kachur R, Bull S, Rietmeijer C. Woman, the Internet, and sexually transmitted infections. Journal of Women's Health 2004; 13 (6): 689-694.

Recibido: 9 de abril 2014; Aceptado: 5 de mayo 2014

ISSN 1695-6141

() COPYRIGHT Servicio de Publicaciones - Universidad de Murcia 